\title{
GEOMETRIC PHASES AND RELATED STRUCTURES ${ }^{\dagger}$
}

\author{
ARMin UhLMANN \\ Mathematical Institute, University of Leipzig \\ Augustusplatz, D-04109 Leipzig, Germany
}

(Received May 26, 1995)

The parallel transport responsible for the geometric phase is reviewed emphasizing the role of transition probabilities and of the metric of Bures.

\section{Introduction}

The phase of a single state is not an observable quantity. In particular, the phase commutes with the observables which define the system. Nevertheless the change of states is generally accompanied by a change of the phase that can be called phase transport. If a state $\omega$ is changed in two different ways to become another state $\omega^{\prime}$, the transport of the phases may yield different phases. 'Then their "difference", the relative phase, may become observable by virtue of the superposition principle. A particular case is the cyclic change, where $\omega$ comes back to itself, and the change of the phase will be compared with that of a "trivial" process, where $\omega$ remains stationary.

All this is obvious, both experimentally and theoretically, for pure states. But it should remain true, to a certain instant, for mixed states: At first, in deviating from the pure to the mixed states, i.e. in going from the extreme part into the inner parts of the state space, coherence and correlations will not be destroyed suddenly but gradually, continuously. Secondly, if embedded in a larger system, the mixed states may be seen as restrictions of pure states. Then some "parts" of the relative phase of a cyclic change in the larger system may become decodable already by observables of the smaller system in which the states appear as mixed ones.

The phase transport and the relative phase consist (at least) of two parts, a dynamical and a geometrical one. The geometric part depends only on the shape of the curve in state space which describes the changes of the system, but not on the time needed for that changes. It is a feature that allows to distinct the geometric phase and its transport from the total phase change.

This remarkable fact also opens a heuristic way to see why the geometric phase survives the adiabatic approximation in which the changes become "infinitely slow".

†The invited lecture to the XXVII Symposium on Mathematical Physics, Torun, 6-9 December, 1994. 
Thus, as seen from to-day, it seems quite natural that the geometric phase firstly appeared within applications of the Born-Oppenheimer approximation, Herzberg and LonguetHiggens [5], Mead and Truhlar [15]. Berry [28], has shown, beside others, the generality of the phenomenon for adiabatically guided Hamiltonians, remarking that the transport condition appears, in the language of mechanics, as an anholonomic constraint. Simon [27], elegantly explained its geometric structure in showing that it is a morphism from the cyclic evolutions, which form a loop group in the state space, into the holonomy group of a natural parallel transport. Because he restricted himself to pure states, that holonomy group is $U(1)$. Then Aharonow and Anandan [34] settled the existence of the geometric phase in every cyclic evolution of pure states, whether adiabatic or not. See also [41].

F. Wilczek and A. Zee [29] have been the first in considering the geometric phase of degenerate eigenstates of a parameter dependent Hamiltonian. Here the state $\omega$ can be described by the projection $P$ onto the subspace of eigenvectors. Choose in the Hilbert space $\mathcal{H}$ any orthoframe $\psi_{1}, \ldots, \psi_{m}$ of length $m$ of eigenvectors. It can be considered as a point of an orthogonal Stiefel manifold which is an $U(n)$-bundle over the Grassmann manifold of projections $P$ of rank $m$. Changing the projections along a curve $C, t \rightarrow$ $P(t)$, calls for a (parallel) transport of the orthoframes, which constitute the fibers over the projections. For a cyclic evolution of the projections, one comes back to the same subspace, and hence to another orthoframe. This latter one is related to the one chosen at the beginning of the evolution by a $U(m)$-transformation $U$, the holonomy of the loop. In $[29]$ the transport condition reads

$$
\left\langle\psi_{j}, \frac{d}{d t} \psi_{k}\right\rangle=0, \quad 1 \leq j \leq m, \quad 1 \leq k \leq m
$$

Physically $U$ has to be regarded as a rclative phase. It is called a geometric phase as it depends only on the oriented loop of the projection operators and not on the velocity with which the system runs through it.

In that scheme the fiber bundle depends on the length of the orthoframes. A reformulation without that unconvenience is as follows: With an auxiliary orthoframe $\varphi_{1}, \ldots, \varphi_{m}$ define the partial isometry $W^{\prime}:=\sum\left|\psi_{j}\right\rangle\left\langle\varphi_{j}\right|$. Then (1) can be expressed by

$$
W^{*} \frac{d}{d t} W^{*}=0 . \quad W^{*} W^{*}=P . \quad \operatorname{rank} P=m .
$$

Indeed, let $C: t \rightarrow P=P(t)$ be a curve of projections of rank $m$ and $C^{\prime}$ a path of orthoframes $\psi_{1}, \ldots, \psi_{m}$ of $P \mathcal{H}$. The path $\tilde{C}: t \rightarrow W=W(t)$ respects (2) if and only if it is of the form $W:=\sum\left|\psi_{j}\right\rangle\left\langle\varphi_{j}\right|$, where the path $C^{\prime}$ fulfils (1) and the auxiliary orthoframe remains unchanged along the path, $W^{*} W=$ const.

If $W$ with $W W^{*}=P$ runs through all lifts of a given curve $C$ of fixed rank projections, parallelity is characterized by

$$
\int\left(\frac{d W}{d t}, \frac{d W}{d t}\right) d t=\operatorname{Min} ! \quad \text { or } \quad \int \sqrt{\left(\frac{d W}{d t}, \frac{d W}{d t}\right)} d t=\operatorname{Min} !
$$




$$
\text { with }\left(W_{1}, W_{2}\right):=\operatorname{Tr} W_{1}^{*} W_{2} .
$$

As a device to transport orthoframes of degenerate eigenvectors Fock [2, appendix] minimizes as in (3) an "energy integral" to obtain (1).

However, a finite rank projection is nothing but a rather special density operator, and the present author could extend [32] the scheme to all density operators: Assume a curve of states is given by a curve of density operators (normalized or not):

$$
t \rightarrow \omega_{t}, \quad \omega_{t}(A)=\operatorname{Tr} D(t) A / \operatorname{Tr} D(t), \quad \operatorname{rank} D(t)=\text { const. }
$$

A lift

$$
t \rightarrow W(t), \quad D(t)=W(t) W(t)^{*}
$$

is called parallel iff

$$
W^{*} \frac{d W}{d t}=\frac{d W^{*}}{d t} W
$$

see also Dąbrowski and Jadczyk [38]. One may regard every $W$ with $W W^{*}=D$ as an amplitude of the state given by $D$, so that two amplitudes of the same state differ by an unobservable unitary (or partial isometric) phase $U, W \rightarrow W U$. Again, transporting the amplitude (the phase) along a closed curve of density operators yields a holonomy which is an observable relative phase.

It has been remarked in 1987 [36] that the parallel condition (6) follows from the variational principle like in (3), which in turn is related to the Riemann metric tied to the Bures distance. We shall come back to this point of view.

The following exposition aims to explain the occurrence of the geometric (Berry) phase within a completely natural, as it seems to me, geometric setting. Looking at rather general state spaces, some parts of the theory concerning pure vector states, which are due to the commutative nature of its U(1) gauge theory, are left aside. There are numerous good papers describing them. Instead, the state space or the cone of positive linear forms equipped with the distance of Bures are viewed as (topological) metric spaces, and as a possible background for defining the transport of amplitudes and phases. The behavior of the metric under actions of stochastic mappings, the Riemann form of the Bures metric, and its distinguished place within a certain class of Riemann metrics (a la Petz) will be discussed. On the other hand the gauge theory associated with the parallel transport and living on *-representations with values in the commutant does not belong to the content of this paper.

The last two sections are devoted to density operators where more explicit expression for various quantities can be derived. In the last section some calculations for two-level systems are shown in the vague hope that the deviations for mixed from the pure state case could be dedected experimentally.

\section{Transition probability, parallelity, and Bures distance}

Let us denote by $\mathcal{A}$ a unital $\mathrm{C}^{*}$-algebra with unit element $e$, and by $\omega$ one of its positive linear forms. If in a unital ${ }^{*}$-representations $\pi$ of $\mathcal{A}$ with representation space 
$\mathcal{H}_{\pi}$ the linear form $\omega$ can be represented as a vector state

$$
\omega(a)=\langle\psi, \pi(a) \psi\rangle, \quad \text { all } a \subset \mathcal{A}, \quad \psi \in \mathcal{H}_{\pi},
$$

the vector $\psi$ is called an amplitude of $\omega$ in $\pi$.

Now let $\omega_{1}$ and $\omega_{2}$ be two positive lincar forms. $\Lambda$ lincar form $\nu$ is called a transition form from $\omega_{1}$ to $\omega_{2}$ if and only if

$$
\left|\nu\left(a^{*} b\right)\right|^{2} \leq \omega_{2}\left(a^{*} a\right) \omega_{1}\left(b^{*} b\right) \quad \text { for all } \quad a, b \in \mathcal{A} .
$$

If $\nu$ is a transition form from $\omega_{1}$ to $\omega_{2}$ then $\nu^{*}$, defined by $\nu^{*}(a)=\bar{\nu}\left(a^{*}\right)$, is a transition form from $\omega_{2}$ to $\omega_{1}$.

Given amplitudes $\psi_{1}, \psi_{2}$ of the positive linear forms $\omega_{1}, \omega_{2}$ in $\pi$, the linear form

$$
a \mapsto \nu(a):=\left\langle\psi_{2}, \pi(a) \dot{\psi}_{1}\right\rangle \quad \text { with } \quad a \in \mathcal{A}
$$

is a transition form from $\omega_{1}$ to $\omega_{2}$. Every transition form can be gained this way. This justifies the interpretation of $\nu(e)$ as a transition amplitude and, if the positive forms are states, of its absolute square $|\nu(e)|^{2}$ as a transition probability. It is tempting to consider the supreme of all transition probabilities reachable by pairs of amplitudes. Thus, if the positive forms are states of the algebra, the supreme of $|\nu(e)|^{2}$ marks the maximally possible transition probability between them. This was the reason in [13] to define the transition probability of two positive linear forms on $\mathcal{A}$ by

$$
P\left(\omega_{1}, \omega_{2}\right):=\sup |\nu(e)|^{2},
$$

where $\nu$ runs through all transition forms from $\omega_{1}$ to $\omega_{2}$. In case the transition probability is not zero, the two states are called overlapping. In turn, vanishing transition probability marks a non-overlapping pair of positive linear forms.

Remark: A correct probability interpretation needs $\omega_{j}(e)=1$. However, the word "transition probability" will be used even if this assumption fails.

The transition probability is symmetric and homogeneous of degree one,

$$
P\left(\omega_{1}, \omega_{2}\right)=P\left(\omega_{2}, \omega_{1}\right), \quad P\left(\lambda_{1} \omega_{1}, \lambda_{2} \omega_{2}\right)=\lambda_{1} \lambda_{2} P\left(\omega_{1}, \omega_{2}\right), \quad \lambda_{j} \geq 0 .
$$

Notice that $P(\omega, \omega)=\omega(e)$, and that $\omega$ is the unique transition form from $\omega$ to $\omega$ with that property.

Let us return to the transition forms. As the definition implies the weak compactness of the set of all transition forms from $\omega_{1}$ to $\omega_{2}$, the supreme of the function $e \rightarrow|\nu(e)|$ is attained on this set. If this happens with $\nu$ it will be called muximal. A transition form $\nu$ is called optimal iff it is maximal and $\nu(e)$ is a not negative real number.

Two amplitudes satisfying (9) with an optimal $\nu$ are called parallel [32].

The transition probability is nicely connected with elementary geometrical properties. Denote by $\psi_{1}, \psi_{2}$ two amplitudes of the positive linear forms $\omega_{1}$ and $\omega_{2}$ in the same representation $\pi$. With $\nu$ defined by (9) one gets for the Hilbert space distance

$$
\left\|\psi_{2}-\psi_{1}\right\|^{2}=\omega_{1}(e)+\omega_{2}(e)-\nu(e)-\nu^{*}(e) .
$$


The shortest possible distance one obviously gets with an optimal $\nu$ or, what is the same, with parallel amplitudes. Then $\nu(e)$ is the square root of the transition probability. This way we arrive at the distance of Bures, dist $_{B}$, see [7],

$$
\operatorname{dist}_{B}\left(\omega_{1}, \omega_{2}\right):=\sqrt{\omega_{1}(c)+\omega_{2}(e)-2 \sqrt{P\left(\omega_{1}, \omega_{2}\right)}} .
$$

Instead of minimizing the Hilbert distance (12) one could have maximized the Hilbert space norm $\left\|\psi_{1}+\psi_{2}\right\|$, which reminds us of the phase convention of Pancharatnam [4]. While Pancharatnam was looking for maximal intensities if two rays of polarized light superpose, Bures asked for a non-commutative version of the Kakutani mean [3]. Though outside the scope of this paper, I mention the appearance of the Bures distance in the theory of "quantum channels" as anticipated by Holevo [19]. Presently we need the metrical aspect to express parallelity.

Before calling (13) a distance one has to check the axioms for a topological metric. The only nontrivial part is in proving the triangle inequality. Starting with three positive linear forms $\omega, \omega_{1}, \omega_{3}$, and optinal transition forms $\nu_{j}$ from $\omega$ to $\omega_{j}$, one considers the GNS-representation $\pi$ of $\omega$ based on the amplitude $\psi$. Because of (8) there are amplitudes $\psi_{1}$ and $\psi_{2}$ such that

$$
\nu_{j}(a):=\left\langle\psi_{j}, \pi(a) \psi\right\rangle, \quad \omega_{j}^{\prime}(a):=\left\langle\psi_{j}, \pi(a) \psi_{j}\right\rangle
$$

with $\omega_{j}^{\prime} \leq \omega_{j}$. The Hilbert space triangle inequality now reads

$$
\sqrt{\omega_{1}^{\prime}(e)+\omega_{2}^{\prime}(e)-2 \Re \nu_{12}(e)} \leq \sqrt{\omega_{1}^{\prime}(e)+\omega(e)-2 \nu_{1}(e)}+\sqrt{\omega_{2}^{\prime}(e)+\omega(e)-2 \nu_{2}(e)},
$$

where $\nu_{12}$ is the transition form from $\omega_{2}^{\prime}$ to $\omega_{1}^{\prime}$ given by the amplitudes $\psi_{j}$. From such an inequality $\sqrt{x} \leq \sqrt{x_{1}}+\sqrt{x_{2}}$ it follows $\sqrt{x+a_{1}+a_{2}} \leq \sqrt{x_{1}+a_{1}}+\sqrt{x_{2}+a_{2}}$ if $a_{j} \geq 0$. Setting $a_{j}=\omega_{j}(e)-\omega_{j}^{\prime}(e)$ we get

$$
\sqrt{\omega_{1}(e)+\omega_{2}(e)-2 \Re \nu_{12}(e)} \leq \operatorname{dist}_{B}\left(\omega_{1}, \omega\right)+\operatorname{dist}_{B}\left(\omega, \omega_{2}\right) .
$$

By definition the left hand side is larger than the Bures distance of the linear forms involved. q.e.d.

Equipped with the distance dist $_{B}$ the cone of positive linear forms is a complete topological metric space. Its metric is an inner one.

The metric is inner because every pair of positive linear forms can be connected by (at least) one curve, called short geodesic arc, the Bures length of which is equal to the Bures distance of the pair.

To prove this one chooses a parallel pair of amplitudes $\psi_{0}, \psi_{1}$ and denotes by $\omega_{t}$ the vector state given by $\psi_{t}=\psi_{0}+t\left(\psi_{1}-\psi_{0}\right)$. For $0<t<1$ one gets

$$
\operatorname{dist}_{B}\left(\omega_{0}, \omega_{t}\right)+\operatorname{dist}_{B}\left(\omega_{t}, \omega_{1}\right) \leq\left\|\psi_{0}-\psi_{t}\right\|+\left\|\psi_{t}-\psi_{1}\right\|=\left\|\psi_{0}-\psi_{1}\right\| .
$$

But the right hand side equals the Bures distance between $\omega_{0}$ and $\omega_{1}$, and must hence be equal to the left hand side because of the triangle inequality. Thus $\psi_{t}$ is parallel to 
$\psi_{0}$ and $\psi_{1}$. The same argument now shows that $\psi_{s}, \psi_{t}$ are parallel for all $0 \leq s \leq t \leq 1$. Thus the Bures length of the curve $t \rightarrow \psi_{t}, 0 \leq t \leq 1$ is equal to the Bures distance of the forms it connects. It remains to prove completeness. Using parallel amplitudes in $\mathcal{H}_{\pi}$ as in the setting above one estimates

$$
\left|\omega_{1}(a)-\omega_{0}(a)\right| \leq\|a\|\left\{\sqrt{\omega_{1}(e)}+\sqrt{\omega_{0}(e)}\right\} \operatorname{dist}_{B}\left(\omega_{1}, \omega_{0}\right) .
$$

Hence the metrical topology is stronger than the $\|\cdot\|_{1}$ topology. q.e.d.

We have seen how an optimal transition form $\nu$ from $\omega_{0}$ to $\omega_{1}$ determines a short geodesic arc by

$$
t \rightarrow \omega_{t}:=(1-t)^{2} \omega_{0}+t^{2} \omega_{1}+t(1-t)\left(\nu+\nu^{*}\right) .
$$

By differentiating and setting $t=0$ we get a linear form $\dot{\omega}_{0}$, the tangent of the curve (15) at $\omega_{0}$,

$$
\dot{\omega}_{0}=\nu+\nu^{*}-2 \omega_{0} .
$$

An obvious question is to be asked: Is there only one short geodesic arc connecting two given states? It follows from (15) that this will occur if the transition form between the initial and final state is uniquely determined. This can happen for overlapping pairs of states only. Sufficient conditions have been proved by Alberti [39] (see also [44]), who uses the word "skew form" to denote certain transition forms. The perhaps most interesting one reads:

A pair $\omega_{1}, \omega_{2}$, of two positive linear forms with equal supports, i.e. annihilating the same left ideals, allows for one and only one optimal transition form from $\omega_{1}$ to $\omega_{2}$.

Let us now look again at the cone of positive linear forms as a topological metric space equipped with the Bures distance. A curve $t \rightarrow \varrho_{t}$ is a continuous map from some interval of the reals into this space. The curve is rectifiable iff it is of finite length. 'l'his length is called Bures length. A curve consisting of finite many short geodesic arcs is a geodesic polygon. A curve of finite length can be metrically approximated by geodesic polygons.

Every subset of a metric space is a metric space again. If in addition every two of its points can be connected by a curve of finite length, the infimum of these length define a new metric which is inner and which is topologically equivalent to the induced relative topology.

This remark applies to the state space $\Omega(\mathcal{A})$, the set of the positive linear forms $\omega$ with $\omega(e)=1$. It becomes a metric space by restricting dist $_{B}$ to it. Clearly, dist $_{B}$ is not an inner metric of the state space. To cure this we start with two states $\omega_{0}, \omega_{1}$ and a pair of its parallel amplitudes $\psi_{0}, \psi_{1}$, in a ${ }^{*}$-representation $\pi$. They mark two point of the unit sphere in $\mathcal{H}_{\pi}$ and generate a real plane in that space. The sphere and the plane intersect through a large circle of the sphere, and there is no shorter curve on the unit sphere than the one on this large circle. Hence there is no curve on the state space connecting $\omega_{0}$ and $\omega_{1}$ with a Bures length shorter than that of $t \rightarrow \omega_{t}(e)^{-1} \omega_{t}$, where $\omega_{t}$ is given by (15) and $\nu$ by the amplitudes $\psi_{0}$ and $\psi_{1}$, see also [52] 'I'herefore, the Bures length of this curve defines an inner metric on the state space. Let us call this distance 
Dist $_{B}$. Elementary geometry tells us

$$
P\left(\omega_{0}, \omega_{1}\right)=\cos ^{2}\left(\operatorname{Dist}_{B}\left(\omega_{0}, \omega_{1}\right)\right), \quad \operatorname{dist}_{B}=2 \sin \frac{1}{2} \operatorname{Dist}_{B}, \quad \operatorname{Dist}_{B} \leq \pi / 2 .
$$

At the manifold of pure states of a type I factor Dist ${ }_{B}$ is the Study-Fubini distance.

\section{Parallel transport, Riemann form of the metric}

We have seen that parallelity of amplitudes takes place iff their Hilbert distance equals their Bures distance dist ${ }_{B}$. Equivalently, one can require that their Study-Fubini distance is measured by Dist ${ }_{B}$. These observations can be interpreted as a parallel transport along short geodesic arcs if the term parallel transport is suitably defined. Before doing so the definition of lifts is necessary.

Let $\gamma: t \rightarrow w_{t}, t_{0} \leq t \leq t_{1}$ be a curve of finite Bures length in the cone of positive linear forms. A lift $\Gamma$ of $\gamma$ is a (strongly continuous) curve $t \rightarrow \psi_{t}$ in a ${ }^{*}$-representation $\pi$ such that every $\psi_{t}$ is an amplitude of $\omega_{t}$ in $\pi$. A lift transports the amplitude and hence the phase from its departure $\psi_{0}$ to its termination $\psi_{1}$. If the base curve $\gamma$ is closed, $\psi_{1}$ will differ from $\psi_{0}$ by a relative phase which is an isometry of the commutant. What can be observed from the point of view of the algebra $\mathcal{A}$ is the transition form defined by the in- and out-vectors: Every lift $\Gamma$ of $\gamma$ defines a transition form

$$
a \mapsto \nu_{\Gamma}(a):=\left\langle\psi_{1}, \pi(a) \psi_{0}\right\rangle
$$

from $\omega_{0}$ to $\omega_{1}$. In case $\gamma$ is a closed loop, $\omega_{1}=\omega_{0}$, the transported and the not transported amplitude may superpose to a new amplitude $\psi_{0}+\psi_{1}$ resulting in a new positive linear form

$$
\frac{1}{2} \omega+\frac{1}{2}\left(\nu_{\Gamma}+\nu_{\Gamma}^{*}\right)
$$

Its value at $a=e$ measures the intensity.

A lift $\Gamma$ of $\gamma$ is called parallel iff for every $t_{0}<t^{\prime} \leq t_{1}$ the Bures length of $t \rightarrow w_{t}$, $t_{0} \leq t \leq t^{\prime}$, is equal to the Hilbert length of the lift $\psi_{t}$ restricted to the same parameter interval $t_{0} \leq t \leq t^{\prime}$. We then write

$$
\nu_{\gamma}:=\nu_{\Gamma}, \quad \Gamma \text { parallel lift of } \gamma,
$$

to indicate that what is visible from $\mathcal{A}$ does not dependent on the choice of the parallel lift. (It is fairly straightforward to prove this for geodesic polygons. A curve with finite Bures length can be approximated by such polygons. To convert this into an approximation of a parallel lift is technically not easy. See the proof of Alberti [45] in a slightly different but similar setting.) If $\gamma$ is closed then the relative phase comparing the initial and the final state is usually called the geometric phase of the loop. The geometric phase of a loop consisting of strictly pure states is Berry's phase factor.

If a parallel lift exists, there is no lift with a Hilbert space length properly smaller than that of the parallel one. This is the minimal length property of parallel lifts. One should notice at this place the possibility of choosing any complete inner topological 
metric on the positive linear forms or on the states, and to define parallelity of lifts just in the same way by requiring the equality of the original and the Hilbert arc length. This may give useful alternatives. But the minimal length property gets lost.

Let us consider a strongly differentiable parallel lift $t \rightarrow \psi_{t}$. Denoting the $t$-derivatives by the dot notation, the square of the line element along the curve is $\langle\dot{\psi}, \dot{\psi}\rangle$. Applying the unitaries $U(t)=\exp i t B$ with a bounded hermitian operator $B$ to the lift, $U(t) \psi_{t}:=\varphi(t)$ one gets another lift of the base curve, provided $B$ commutes with $\pi(\mathcal{A})$. The minimal length property results in

$$
\langle\dot{\psi}, \dot{\psi}\rangle \leq\langle\dot{\varphi}, \dot{\varphi}\rangle,
$$

which may be rewritten as

$$
0 \leq\langle i B \psi, \dot{\psi}\rangle+\langle\dot{\psi}, i B \psi\rangle+\langle B \psi, B \psi\rangle .
$$

This can only be valid for all real multiples of $B$ iff the parallelity condition

$$
\langle\psi, B \dot{\psi}\rangle=\langle\dot{\psi}, B \psi\rangle, \quad B \in \pi(\mathcal{A})^{\prime}
$$

is true for all hermitian and hence for all elements of the commutant $\pi(\mathcal{A})^{\prime}$. This is equivalent with the statement that every curve $t \rightarrow B_{t} \psi_{t}$ with $B_{t}$ in the commutant fulfils Berry's transport condition [28]. In the situation considered in the introduction, equations (6) and (22) are equivalent.

The equations (22) give necessary conditions for the tangent $\dot{\psi}$ at $\psi$. Dąbrowski and Jadczyk [38] had the idea to solve them by an ansatz

$$
\dot{\psi}=\pi(g) \psi, \quad g=g^{*} \in \mathcal{A} .
$$

Let us call these tangents regular. They form a real linear submanifold of $\mathcal{h}_{\pi}$. The latter becomes a real pre-Hilbert space by

$$
2\left\langle\varphi_{1}, \varphi_{2}\right\rangle^{\text {real }}:=\left\langle\varphi_{1}, \varphi_{2}\right\rangle+\left\langle\varphi_{2}, \varphi_{1}\right\rangle,
$$

so that for two regular tangents $\dot{\psi}_{j}$ at $\psi$

$$
2\left\langle\dot{\psi}_{1}, \dot{\psi}_{2}\right\rangle^{\text {real }}:=\left\langle\psi,\left(g_{1} g_{2}+g_{2} g_{1}\right) \psi\right\rangle
$$

The closure of the space of real tangents becomes a real Hilbert subspace, the space of horizontal tangents $\mathcal{T}_{\psi}^{\text {hor }}$. Its real orthogonal complement $\mathcal{T}_{\psi}^{\text {ver }}$ consists of the vertical tangents. Thus the tangent space of the representation space, seen as real Hilbert space, splits at every vector different from 0 in an horizontal and a vertical subspace. The real Hilbert scalar product on every fiber $\mathcal{T}_{\psi}$ of the tangent space is a real Riemann structure. (If $\psi$ is the GNS vacuum of a faithful normal $\omega$ of a von Neumann algebra, the tangents (23) point into Araki's 0-cone $[9,10]$ based at $\psi$.)

Going back to the algebra, equation (23) is equivalent with

$$
\dot{\omega}(a)=\omega(a g+g a), \quad g=g^{*}, \quad \forall a \in \mathcal{A} .
$$


A linear form $\dot{\omega}$ allowing for a representation $(26)$ is called a regular tangent at $\omega$. According to Sakai, [6] and [8], proposition 1.24.4, $g$ exists if in case of a normal state $\omega$ of a $\mathrm{W}^{*}$-algebra $\dot{\omega}$ can be written as the difference of two positive forms both of which are dominated by $\omega$.

Though the element $g$ is not necessarily unique,

$$
(\dot{\omega}, \dot{\omega})_{\omega}:=\frac{1}{2} \dot{\omega}(g)=\omega\left(g^{2}\right), \quad d_{\mathrm{B}} s=\sqrt{(\dot{\omega}, \dot{\omega})} d t
$$

is uniquely associated to a given regular tangent. It extends to a real positive definite scalar product. The closure $\mathcal{T}_{\omega}$ of the real linear space of all regular tangents with respect to it is the tangent space at $\omega$, and the real quadratic form (27) extended to the whole tangent space defines a Riemannian metric form at $\omega$. It is the Riemannian metric belonging to the Bures distance dist ${ }_{B}$, and $d_{\mathrm{B}} s$ is its line element at $\omega$. See also [51]. As a so-called statistical distance (27) appears (for matrix algebras) in [55].

For every $\omega$ and any of its amplitudes $\psi$ the correspondence between $\pi(g) \psi$ and $\dot{\omega}(a):=\omega(a g+g a)$ induces an isometry between $\mathcal{T}_{\omega}$ and $\mathcal{T}_{\psi}^{\text {hor }}$.

The different fibers of the tangent space are not on equal footing but depend on the support and further properties of the base point $\omega$. This expresses the fact we are considering "manifolds with boundaries".

By (27) the state space becomes a Riemannian submanifold with the distance function $\operatorname{Dist}_{B}$, while a tangent at a state $\omega$ has to satisfy the subsidiary condition $\dot{\omega}(e)=0$. The costraint is solved by the map $\dot{\omega} \rightarrow \dot{\omega}-\dot{\omega}(e) \omega$.

The quadratic form which reduces to (27) on the state space, and which is invariant against rescaling $\omega \rightarrow \lambda \omega$ with real and positive functions $\lambda=\lambda(\omega)$, reads

$$
\frac{(\dot{\omega}, \dot{\omega})_{\omega}}{\omega(e)}-\frac{1}{4}\left(\frac{\dot{\omega}(e)}{\omega(e)}\right)^{2}
$$

On the pure vector states both, (27) and (28), encodes the Study-Fubini metric.

\section{Examples, stochastic maps}

Let us assume that the following relation holds:

$$
\omega_{j}(a)=\omega\left(c_{j}^{*} a c_{j}\right), \quad j=1,2, \quad \forall a \in \mathcal{A} .
$$

with elements $c_{1}, c_{2}$ and a positive linear forms $\omega$. The Schwarz inequality shows

$$
P\left(\omega_{1}, \omega_{2}\right) \geq\left|\omega\left(c_{1}^{*} c_{2}\right)\right|^{2}, \quad \text { and } \quad \nu(a)=: \omega\left(c_{1}^{*} a c_{2}\right)
$$

is a transition form from $\omega_{1}$ to $\omega_{2}$. If

$$
c_{1}^{*} c_{2}=c_{2}^{*} c_{1} \geq 0
$$

then $\nu$ as defined by $(30)$ is optimal $[8,9,13]$. 
Let us look only at the proof under the simplifying assumption of invertibility of $c_{1}$. Defining

$$
c:=c_{2} c_{1}^{-1}=\left(c_{1}^{*}\right)^{-1}\left(c_{1}^{*} c_{2}\right) c_{1}^{-1}>0
$$

one gets

$$
\omega\left(c_{1}^{*} c_{2}\right)=\omega_{1}(c)=\omega_{2}\left(c^{-1}\right)
$$

whose square is, according to (30), a lower bound of the transition probability. But it is also an upper bound: Substituting $a$ and $b$ in (8) by the positive square roots of $c$ and of $c^{-1}$ is showing this, q.e.d. In the case just considered with $c$ as in (32) the comparison with (15) and (16) proves the element $g:=c-e$ to be the regular tangent at $\omega_{1}$ of the short geodesic arc connecting $\omega_{1}$ with $\omega_{2}$.

The statement above can be used to parallel transport along certain geodesic polygons. Let $\omega_{1}, \omega_{2}, \ldots, \omega_{n}$ be an ordered set of states (or positive linear forms). Assume $\omega_{1}()=$. $\omega\left(c_{1}^{*} \cdot c_{1}\right)$ and the possibility of choosing inductively $c_{j+1}$ by $\omega_{j+1}=\omega\left(c_{j+1}^{*} \cdot c_{j+1}\right)$ and $c_{j}^{*} c_{j+1}>0$. The optimal transition forms $\omega\left(c_{j}^{*} \cdot c_{j+1}\right)$ then define a geodesic polygon, $\gamma$, and the result of the parallel transport along $\gamma$ equals $\nu_{\gamma}:=\omega\left(c_{1}^{*} \cdot c_{n}\right)$.

Quite another interesting application is to unital commutative $\mathrm{C}^{*}$-algebras $\mathcal{F}$. Being canonically isomorphic to some algebra $\mathcal{C}(X)$ of continuous functions on a compact $X$, the states are indexed by measures: The expectation value $\omega(a)$ is the integral of the function $a$ on $X$ over a measure $d \mu$. Denoting by $c_{1}, c_{2}$ the positive roots of the RadonNikodym derivatives of two measures, $d \mu_{1}, d \mu_{2}$, with respect to $d \mu$, the condition (31) is trivially fulfilled. The optimal transition form $a \rightarrow \omega\left(c_{1} c_{2} a\right)$ is the Kakutani mean. With $a=e$ one obtains Kakutani's invariant which is nothing but $P\left(\omega_{1}, \omega_{2}\right)$, where the states $\omega_{j}$ are defined by the measures $d \mu_{j}$. Starting from this statement it is possible to identify the Bures distance Dist ${ }_{B}$ with the distance function of what is now called Fisher metric on measure spaces. In Fisher's paper [1] the commutative version of the the quadratic form (27) is assumed to measure the "intrinsic accuracy of an error curve". Explicit expressions on the simplex of probability vectors are due to Wotters [18], who also noticed similarities with the Study-Fubini metric. An independent proof that Dist ${ }_{B}$ fulfils the triangle inequality on measure spaces has been given in [30].

It is Cantoni's idea [12] to apply Kakutani's procedure in order to get a transition probability between two states of a suitable algebra. Starting with a pair of states he defined their transition probability to be the supreme of the Kakutani invariants of their restrictions to all maximal commutative unital *-subalgebras, see also [23]. Araki and Raggio [21], see also [22], proved for normal states of von Neumann algebras the equivalence of Cantoni's definition with the one given by (10). Their results could be extended by Alberti [24] and, in other directions, by Gudder, Marchand, and Wyss [14], and Kosaki [26].

Alberti proved the validity of

$$
P(\omega, \varrho)=\inf _{b} \omega(b) \varrho\left(b^{-1}\right), \quad b>0, \quad b, b^{-1} \in \mathcal{A}
$$

for any two positive linear forms $\omega, \varrho$ of a unital $\mathrm{C}^{*}$-algebra. (Notice (33) as a particular case.) Because $b$ and $b^{-1}$ obviously commute, (34) is a sharpening of Cantoni's procedure. 
Let us now turn to mappings. A stochastic map $\phi$ is the dual of a positive unital map $\phi_{*}$ mapping a unital *-algebra $\tilde{\mathcal{A}}$ into another one $\mathcal{A}$. It maps positive linear forms as well as states of $\mathcal{A}$ into those of $\tilde{\mathcal{A}}$ according to

$$
\phi: \omega \mapsto \omega \circ \phi=\tilde{\omega}, \quad \tilde{\omega}(\tilde{a}):=\omega\left(\phi_{*}(\tilde{a})\right)
$$

Applying this notation to $(34)$ with a positive and invertible element $\tilde{b}$ of $\tilde{\mathcal{A}}$, one gets by an inequality of Choi [16]

$$
P(\omega, \varrho) \leq \omega\left(\phi_{*}(\tilde{b})\right) \varrho\left(\phi_{*}(\tilde{b})^{-1}\right) \leq \omega\left(\phi_{*}(\tilde{b})\right) \varrho\left(\phi_{*}\left(\tilde{b}^{-1}\right)\right)
$$

Taking the infimum with respect of $\tilde{\mathcal{A}}$ as required by Alberti's relation one arrives at [25]

$$
P(\omega \circ \phi, \varrho \circ \phi) \geq P(\omega, \varrho)
$$

Thus the transition probability is increasing and, by (13), (17), the Bures distances dist ${ }_{B}$ and Dist $_{B}$ are contracting under the action of a stochastic map.

A particular case of a stochastic map is the restriction of the positive forms onto a unital $\mathrm{C}^{*}$-subalgebra, i.e. the dual of a unital inclusion map. The condition (8) is less stringent if only the elements of a subalgebra are at our disposal. Hence (35) is rather elementary for restrictions. We shall ask for functors associating to every pair $\omega_{1}, \omega_{2}$ of every unital $\mathrm{C}^{*}$-algebra a real number $\hat{P}\left(\omega_{1}, \omega_{2}\right)$ such that $\hat{P}$ is increasing under the action of restriction maps. One can relate some of these objects to the transition probability, and this is the occasion to introduce the following.

A state $\omega$ of $\mathcal{A}$ is called strictly pure iff there is a projection $p \in \mathcal{A}$ such that

$$
\forall a \in \mathcal{A}: \quad p a p=\omega(a) p, \quad p=p^{*}=p^{2} \in \mathcal{A},
$$

and one says $p$ implements $\omega$. With every overlapping positive linear form $\varrho$ one obtains the optimal transition form

$$
a \rightarrow \nu(a)=\frac{\varrho(a p)}{\sqrt{\varrho(p)}}
$$

from $\omega$ to $\varrho$. The reasoning is as from (29) to $(31)$, for instance with $c_{1}=e$ and $c_{2}=p$. Thus

$$
P(\omega, \varrho)=\varrho(p), \quad p \text { implements } \omega
$$

If (37) exists, i.e. if $\varrho$ and the strictly pure state $\omega$ are overlapping, then there is no other optimal transition form than that given by (37), [31].

Let us return to an arbitrary pair of states, $\omega_{1}, \omega_{2}$ and let us chose parallel amplitudes $\psi_{1}, \psi_{2}$ for them in a unital and faithful ${ }^{*}$-representation $\pi$. In $\mathcal{B}=\mathcal{B}\left(\mathcal{H}_{\pi}\right)$ these amplitudes define strictly pure states, $\varrho_{1}, \varrho_{2}$, and $\pi$ embeds $\mathcal{A}$ into $\mathcal{B}$. Because of the assumed parallelity,

$$
P\left(\omega_{1}, \omega_{2}\right)=P\left(\varrho_{1}, \varrho_{2}\right), \quad \omega_{j}=\varrho_{j} \circ \pi^{*}
$$


Assume a functor $\hat{P}$, defined on pairs of states of every unital $\mathrm{C}^{*}$-algebra, is increasing under restrictions and coincides with $P$ for pairs of strictly pure states. Then

$$
P\left(\omega_{1}, \omega_{2}\right)=P\left(\varrho_{1}, \varrho_{2}\right)=\hat{P}\left(\varrho_{1}, \varrho_{2}\right) \leq \hat{P}\left(\omega_{1}, \omega_{2}\right) .
$$

Every functor $\hat{P}$ on pairs of states, increasing under restrictions and coinciding with $P$ for pairs of strictly pure states, satisfies $P \leq \hat{P}$.

Further, assume on every state space a distance Dist is given, contracting under restriction maps and coinciding with Dist $_{B}$ on pairs of strictly pure states. Then Dist $\leq$ Dist $_{B}$. If Dist can be gained from a Riemann metric, its line element will be smaller than Bures's one.

There are several variants of these statements. For example, assume $\hat{P}$ is defined for pairs of normal states on $\mathcal{B}(\mathcal{H})$ and $\mathcal{H}$ is not finite dimensional. If $\hat{P}$ coincides with $P$ on pairs of normal pure states and if $\hat{P}$ is increasing for stochastic cp-endomorphisms, then $\hat{P} \geq P$ for pairs of normal states. (cp means complete positive.) Similarly Dist ${ }_{B}$ can be characterized on the set of normal states of an infinite type I factor.

New questions arise in asking for Riemann structures defined on the set of faithful states and contracting under stochastic mappings. For finite ${ }^{*}$-matrix algebras Petz [56], see also [57], relying on the classification of operator means by Kubo and Ando [17] has been able to classify them. On commutative (sub)algebras they reduce, up to a numerical factor, to the Fisher metric. This has to be so by the Cencov uniqueness theorem [20, 33]. To get rid of the above mentioned factor, Petz demands equality with the Fisher metric on finite commutative unital ${ }^{*}$-subalgebras. (For full matrix algebras it suffices to have equality at the tracial state.)

The class of metrics described by Petz can be analysed in terms of its extremal members. In Petz's classification scheme they belong to the operator monotone functions $f(x)=s x+1-s$. We define them on their complexified tangent spaces over the cone of positive linear forms of a general $\mathrm{C}^{*}$-algebra,

Choosing first for our task a real number $s$ from the unit interval, a linear form $\nu$ is called a regular complex tangent on the positive linear form $\omega$ if there is $g \in \mathcal{A}$ such that

$$
\nu(a)=2(1-s) \omega(a g)+2 s \omega(g a), \quad a \in \mathcal{A} .
$$

For $s=1 / 2$ and hermitian $g$ we fall back to the Bures case (26). With two tangents $\nu_{j}$ of (39) induced by the elements $g_{1}, g_{2}$ we perform

$$
\left(\nu_{1}, \nu_{2}\right)_{\omega}^{s}:=(1-s) \omega\left(g_{1}^{*} g_{2}\right)+s \omega\left(g_{2} g_{1}^{*}\right)
$$

so that

$$
\left(\nu_{1}, \nu_{2}\right)_{\omega}^{s}=\frac{1}{2} \nu_{2}\left(g_{1}^{*}\right)=\frac{1}{2} \nu_{1}^{*}\left(g_{2}\right)=\left(\nu_{2}^{*}, \nu_{1}^{*}\right)_{\omega}^{1-s} .
$$

There may be different $g$ in (39) resulting in the same tangent. However, $(40)$ is independent of that arbitrariness and it defines a positive definite scalar product on the regular tangents. The completion of the regular complex tangents with respect of that scalar product is denoted by $\mathcal{C T}_{\omega}^{s}$ and called the complex s-tangent space at $\omega$. The completion 
can and will be performed within $\mathcal{A}^{*}$. This setting defines a Riemannian metric on the space $\mathcal{C T}^{s}$ of complex $s$-tangents. The map $\nu \rightarrow \nu^{*}$ is an isometric conjugation from $\mathrm{CT}^{s}$ onto $\mathcal{C T}^{1-s}$ as one can see from (41). For $s=1 / 2$, the Bures case, the complex tangent space is self-conjugate.

The class of metrics described by Petz is obtained through

$$
\left(\nu_{1}, \nu_{2}\right)_{\omega}^{(d \mu)}:=\int\left(\nu_{1}, \nu_{2}\right)_{\omega}^{s} d \mu(s)
$$

where $d \mu$ is a measure on the unit interval. On commutative *-algebras every one is a multiple of the Fisher metric. To get equality $d \mu$ has to be a probability measure. We then call (42) Fisher adjusted.

In type I factors the extremal metrics (41) are tied together by the harmonic mean interpolation

$$
(\nu, \nu)_{\omega}^{s}=\left(\frac{1+t}{t}\right) \inf \left[t\left(\nu_{1}, \nu_{1}\right)_{\omega}^{s^{\prime}}+\left(\nu_{2}, \nu_{2}\right)_{\omega}^{s^{\prime \prime}}\right]
$$

with $s^{\prime}$ and $s^{\prime \prime}$ fixed to give

$$
s=\frac{1}{1+t} s^{\prime}+\frac{t}{1+t} s^{\prime \prime},
$$

while the infimum runs through all $\nu_{j}$ such that $\nu=\nu_{1}+\nu_{2}$.

While the proof is rather straightforward for a faithful $\omega$ representable by a density operator, at the time being I do not know a technically complete proof in the general case of unital $\mathrm{C}^{*}$-algebras.

The metrics (42) for which $\nu \rightarrow \nu^{*}$ is an involution induce a real Riemann metric on the real tangent space. In terms of $(42)$ this means $d \mu(s)=d \mu(1-s)$. Restricting ourselves to the cases of validity let us use in (43) and (44) the values $s=1 / 2, t=1$, $s^{\prime}=s^{\prime \prime}=k$, and the tangents $\nu_{j}=(1 / 2) \nu$. We get

$$
(\nu, \nu)_{\omega} \leq \frac{1}{2}\left[(\nu, \nu)_{\omega}^{k}+(\nu, \nu)_{\omega}^{1-k}\right] .
$$

On the left hand side one identifies the metric of Bures, the metrics of the right hand side are the extremal ones within the real metrics of (42). Thus we have gone another way to see a conclusion of Petz, which we now combine with estimates above:

Let a real metric be given by (42). If it is Fisher adjusted then its line element is never smaller than that of the Bures metric. If it is Study-Fubini adjusted, (which is not always possible), then its line element is never larger than that of Bures. The metric of Bures is the only one in the considered class which is simultaneously adjusted to the Fisher and to the Study-Fubini metric.

According to Petz $[56,57]$ the metric forms (42) exhaust all (real or not) monotone Riemann metrics on the manifold of faithful states of the algebras $\mathcal{M}_{n}$. Monotonicity means the action of completely positive stochastic maps is metrically contractive. In our setting monotonicity can be seen rather easily (see below). However, that one gets, under cleverly chosen assumptions, "all" monotone Riemann metrics in the general case is tempting but an open question. 
Indeed, with a completely positive unital map $\phi_{*}$ from $\tilde{\mathcal{A}}$ into $\mathcal{A}$ and a fixed $0 \leq s \leq 1$ one associates

$$
\tilde{\omega}=\omega \circ \phi, \quad \nu_{\phi}(\tilde{a})=2(1-s) \tilde{\omega}(\tilde{a} \phi(g))+2 s \tilde{\omega}(\phi(g) \tilde{a})
$$

for a tangent $\nu$ given by (39). Now, as an immediate consequence of the Kadison inequalities, one gets from (40)

$$
(\nu, \nu)_{\omega}^{s} \geq\left(\nu_{\phi}, \nu_{\phi}\right)_{\tilde{\omega}}^{s} .
$$

Hence all the metrics (42) are monotone (contracting) like in (46).

Remark: Petz classifies the monotone metrics by an operator monotone function $f$. In terms of our definition (42) this function reads

$$
f(x)^{-1}=f_{P}(x)^{-1}=\int \frac{1}{(1-s) x+s} d \mu(s) .
$$

To every contracting metric (42) there is a parallel transport by requiring coincidence of the line element on a path of states with the Hilbert line element along its lift into a unital *-representation. Interesting enough, those transports were all described, for matrix algebras, by Dittmann and Rudolph [46]. In their paper, another function - let me call it $f_{D R}$ - characterizes the different parallel transports. Recently J. Dittmann [58] discovered the relation between $f_{P}$ and $f_{D R}$.

\section{Density operators}

We now simplify the treatment considerably assuming the algebra $\mathcal{A}$ to be isomorphic to $\mathcal{B}(\mathcal{H})$, that is to the algebra of bounded operators acting on a Hilbert space $\mathcal{H}$. Since $\mathcal{B}(\mathcal{H})$ is an operator algebra, we denote its elements as usual by capital letters.

Before we exclusively consider normal states expressible by density operators let us consider a singular state $\varrho$ and a normal state $\omega$. Denoting by $1_{\mathrm{id}}$ the unit element, by $Q$ a finite rank projection, by $\lambda$ a positive real, and abbreviating $B=1_{\mathrm{id}}+\lambda Q$,

$$
\varrho(B)=1, \quad \omega\left(B^{-1}\right)=1-\frac{\lambda}{1+\lambda} \omega(Q) .
$$

Now Alberti's estimation (34) shows

$$
P(\omega, \varrho) \leq 1-\omega(Q),
$$

$Q$ being an arbitrary finite projection operator. Hence,

$$
P\left(\omega_{\text {normal }}, \varrho_{\text {singular }}\right)=0
$$

so that no normal positive form overlaps with any singular one.

From now let us restrict ourselves to normal positive linear forms, respectively states. To express the Bures distance with the help of positive trace class operators we consider three states

$$
\omega(A)=\operatorname{Tr} A D, \quad \omega_{j}(A)=\operatorname{Tr} A D_{j}, \quad A \in \mathcal{B}(\mathcal{H})
$$


where $j=1,2$. Given the last two states we can choose the first in such a way that we meet the situation assumed in (29) with bounded operators $C_{j}$. These operator are uniquely determined by requiring $C_{j} \psi=0$ if $D \psi=0$. It is convenient to introduce the Hilbert Schmidt operators

$$
W_{j}:=C_{j} D^{1 / 2} \quad \text { obeying } \quad W_{j} W_{j}^{*}=D_{j} .
$$

To get an optimal transition form from $\omega_{1}$ to $\omega_{2}$ we have to ensure, according to (31), the positivity condition

$$
C_{1}^{*} C_{2} \geq 0 \quad \text { or } \quad W_{1}^{*} W_{2} \geq 0 .
$$

The positivity enables us to write

$$
W_{1}^{*} W_{2}=\sqrt{W_{1}^{*} W_{2} W_{2}^{*} W_{1}}=\sqrt{W_{2}^{*} W_{1} W_{1}^{*} W_{2}} .
$$

From (51) and the polar decompositions

$$
W_{j}=D_{j}^{1 / 2} V_{j}, \quad j=1,2,
$$

we conclude

$$
W_{1}^{*} W_{2}=V_{1}^{*} \sqrt{D_{1}^{1 / 2} D_{2} D_{1}^{1 / 2}} V_{1}=V_{2}^{*} \sqrt{D_{2}^{1 / 2} D_{1} D_{2}^{1 / 2}} V_{2} .
$$

Because of parallelity (30) together with (54) shows

$$
\begin{gathered}
P\left(D_{1}, D_{2}\right):=P\left(\omega_{1}, \omega_{2}\right)=\omega\left(C_{1}^{*} C_{2}\right)=\operatorname{Tr} W_{1}^{*} W_{2}, \\
P\left(D_{1}, D_{2}\right)=\operatorname{Tr} \sqrt{D_{1}^{1 / 2} D_{2} D_{1}^{1 / 2}}=\operatorname{Tr} \sqrt{D_{2}^{1 / 2} D_{1} D_{2}^{1 / 2}},
\end{gathered}
$$

as well as the optimal transition amplitude from $\omega_{1}$ to $\omega_{2}$

$$
\nu(A)=\omega\left(C_{1}^{*} A C_{2}\right)=\operatorname{Tr} A W_{2} W_{1}^{*} .
$$

The polar decomposition (53) applied to (52) provides us with

$$
D_{1}^{1 / 2} W_{2} W_{1}^{*}=\sqrt{D_{2}^{1 / 2} D_{1} D_{2}^{1 / 2}} D_{1}^{1 / 2} .
$$

In order to get not too involved with support discussions, let us assume faithfulness (or equal supports) of our density operators. We then see explicitly how Alberti's uniqueness theorem works. Abbreviating the Pusz and Woronowicz geometric mean [11] by ( $S, R$ positive)

$$
S \# R:=R^{1 / 2}\left(R^{-1 / 2} S R^{-1 / 2}\right)^{1 / 2} R^{1 / 2},
$$

(58) tells us

$$
W_{2}=\left(D_{2} \# D_{1}^{-1}\right) W_{1} .
$$

Taking into account (53) one can define a relative phase by

$$
W_{2}=D_{2}^{1 / 2} U_{2,1} V_{1}, \quad U\left(\omega_{2}, \omega_{1}\right)=U\left(D_{2}, D_{1}\right):=U_{2,1}
$$


so that $(58)$ or $(60)$ yields

$$
U_{2,1}=D_{2}^{-1 / 2} D_{1}^{-1 / 2}\left(D_{1}^{1 / 2} D_{2} D_{1}^{1 / 2}\right)^{1 / 2} .
$$

This phase depends only on the ordered pair of states (density operators).

In finite dimensions and for faithful states the unitaries (62) are expressed through products of positive operators. Therefore, their determinants have to be one:

$$
\operatorname{det} U_{2,1}=\operatorname{det} U\left(D_{2}, D_{1}\right)=1, \quad U \in \mathrm{SU}(n) .
$$

The reduction of these relative phases to $S U(n)$ in the interior of the state space is due to Dittmann and Rudolph [47], and to Alberti [45].

An ordered finite set of faithful density operators (states) determine a geodesic polygon

$$
D_{1}=D_{n+1} \Leftarrow D_{n} \Leftarrow \ldots \Leftarrow D_{2} \Leftarrow D_{1}
$$

in the state space. Then the amplitudes and phases are parallel transported according to

$$
W_{1} U:=W_{n+1}=\left(D_{1} \# D_{n}^{-1}\right)\left(D_{n} \# D_{n-1}^{-1}\right) \ldots\left(D_{2} \# D_{1}^{-1}\right) W_{1}
$$

or, using the notations (61) and (62), according to

$$
W_{1} U=W_{n+1}=D_{1}^{1 / 2} U_{n+1, n} U_{n, n-1} \ldots U_{2,1} V_{1} .
$$

The transport condition (6) allows parallel transport for lifts of arbitrary (regular enough) curves of density operators. SU(2)-orbits [40] and, with less details, further Hamiltonian motions $[43,53]$ have been considered.

The parallel transport as determined by the Bures metric is governed by a gauge theory $[43,50]$. Its connection $\mathbf{A}$ form is given by

$$
W^{*} \mathrm{~d} W-\mathrm{d} W^{*} W=W^{*} W \mathbf{A}+\mathbf{A} W^{*} W .
$$

The case of dimension two is well described by Dittmann and Rudolph [47]. Here $\mathbf{A}$ is a connection on the 7-sphere with a 3-semisphere as base space. Not much is known for $n>2$.

The Riemann form of the Bures metric and of the metrics of Petz can be gained as following. A bounded operator $X$ is a regular (complex) tangent of (40) iff there is $G^{(s)}$ such that

$$
X=2(1-s) G^{(s)} D+2 s G^{(s)} D .
$$

'T'hen

$$
(Y, X)_{\omega}^{s}=\frac{1}{2} \operatorname{Tr} Y^{*} G^{(s)},
$$

where $D$ is the density operator of $\omega$. Remind the Bures metric which one gets with $s=1 / 2$. Relying now on the representation (42) one gets

$$
(Y, X)_{\omega}^{d \mu}=\frac{1}{2} \operatorname{Tr} Y^{*} G^{(d \mu)}, \quad G^{(d \mu)}=\int G^{(s)} d \mu .
$$


In this large area of interest let us look only at the extreme boundary, where

$$
D=|\psi\rangle\langle\psi|, \quad X=| \dot{\psi}\rangle\langle\psi|+| \psi\rangle\langle\dot{\psi}| .
$$

Adopting the subsidiary condition $\langle\psi \mid \dot{\psi}\rangle=0$ one gets

$$
2 G^{(s)}=\frac{1}{1-s}|\dot{\psi}\rangle\left\langle\psi\left|+\frac{1}{s}\right| \psi\right\rangle\langle\dot{\psi}|
$$

Hence we get for the submanifold of strictly pure states

$$
\frac{1}{2} \operatorname{Tr} X^{*} G^{(s)}=\frac{1}{4} \frac{1}{s(1-s)}\langle\dot{\psi} \mid \dot{\psi}\rangle
$$

The factor on the right hand side is 1 for the Bures metric only, otherwise it is larger than 1. In order to adjust a general metric (42) to the Study-Fubini metric on the extreme boundary, one has to divide the Fisher adjusted metric by the factor

$$
\int \frac{d \mu(s)}{4 s(1-s)}, \quad \int d \mu(s)=1 .
$$

This is only possible if that factor is finite. The finiteness condition shows which of the considered metrics can be extended to the pure states.

\section{Two-level systems}

The 2-dimensional case allows for an explicit treatments [42] which could not be achieved for higher dimensions yet.

But there are further reasons to embark on it. Indeed, to the first experiments [35] establishing Berry's phase belong configurations with mirrors or mirror-like devices which can be described by geodesic polygons on the 2-sphere of pure states (with diameter one if Study-Fubini adjusted). If one respects helicity reversals by an extra $i \pi$ phase, the polygon can be thought of as consisting of short geodesic arcs. These and similar situations (use of filters) with "quantum jumps" are clearly examined in [37]. An experimental check of parallel transport of amplitudes and phases within the region of mixed states could perhaps become possible in two-level systems. The experimental difficulty is in the accuracy within which a degree of polarization (as an equivalent of a temperature) can be adjusted.

Two-by-two density operators may be described by

$$
D=\frac{1}{2} 1_{\mathrm{id}}+x_{1} \sigma_{1}+x_{2} \sigma_{2}+x_{3} \sigma_{3}=\frac{1-\xi}{2} 1_{\mathrm{id}}+\xi Q,
$$

where $Q$ is a rank one projection and $\left\{2 x_{1}, 2 x_{2}, 2 x_{3}\right\}$ is the polarization vector. Its length $\xi$ is the degree of polarization

$$
\xi^{2}=4\left(x_{1}^{2}+x_{2}^{2}+x_{3}^{2}\right), \quad 4 x^{2}:=\operatorname{det} D=1-\xi^{2} .
$$


The transition probability in (10) can be computed to $[48,49]$

$$
P\left(D, D^{\prime}\right)=\frac{1}{2}+2\left(x_{1} x_{1}^{\prime}+x_{2} x_{2}^{\prime}+x_{3} x_{3}^{\prime}+x x^{\prime}\right) .
$$

One can establish in a straightforward way

$$
D^{\prime} \# D^{-1}==\frac{D^{\prime}+x^{\prime} x D^{-1}}{\sqrt{P\left(D^{\prime}, D\right)}}
$$

and for the relative phases $(65),(66)$ one gets

$$
U\left(D^{\prime}, D\right)=\frac{(D)^{1 / 2}\left(D^{\prime}\right)^{1 / 2}+x x^{\prime}(D)^{-1 / 2}\left(D^{\prime}\right)^{-1 / 2}}{\sqrt{P\left(D^{\prime}, D\right)}} .
$$

This is valid for all short geodesics of length smaller than $\pi / 2$, i.e. if $P\left(D^{\prime}, D\right) \neq 0$. The numerator in the equation above is a polynomial in the matrix elements of $\sqrt{D}$ and of $\sqrt{D^{\prime}}$. Hence $(79)$ is well defined for all pairs of overlapping states.

A particularly simple case appears if the degree of polarization does not change, $\xi=\xi^{\prime}, x=x^{\prime}$. Then

$$
\sqrt{P\left(D^{\prime}, D\right)} D^{\prime} \# D=1_{\text {id }}+D^{\prime}-D=1_{\text {id }}+\xi\left(Q^{\prime}-Q\right) .
$$

As an application, let us compute the geometric phase $U$ of geodesic triangles and quadrangles consisting of short geodesic arcs. Let us denote, as in (75), the common degree of polarization by $\xi$, and the projections of the involved density operators $D_{j}$ by $Q_{j}=|j\rangle\langle j|$. We abbreviate

$$
a_{12}:=\langle 1 \mid 2\rangle\langle 2 \mid 1\rangle, \quad a_{321}:=\langle 3 \mid 2\rangle\langle 2 \mid 1\rangle\langle 1 \mid 3\rangle, \quad \text { and so on . }
$$

\section{Geodesic triangles:}

The expression relevant for the intensities is

$$
\begin{gathered}
\sqrt{a_{12} a_{23} a_{31}} \operatorname{Tr} D_{1} U=\xi^{4} a_{321}+\xi^{3}(1-\xi) \frac{a_{12}+a_{23}+a_{31}+2 a_{321}-3}{2} \\
+\xi^{2}(1-\xi)^{2} \frac{a_{12}+a_{23}+a_{31}+6}{2}+4 \xi(1-\xi)^{3}+(1-\xi)^{4} .
\end{gathered}
$$

For $\xi=1$ on gets $\epsilon=a_{321} /\left|a_{321}\right|$ which is Berry's phase factor of the cyclic process $Q_{1}, Q_{2}, Q_{3}, Q_{1}$ as it should be.

\section{Geodesic quadrangles:}

$$
\begin{gathered}
\sqrt{a_{12} a_{23} a_{34} a_{41}} \operatorname{Tr} D_{1} U=\xi^{5} a_{4321}+\frac{\xi^{4}(1-\xi)}{2}\left(6 a_{4321}-a_{432}-a_{421}+3 a_{431}\right. \\
\left.+3 a_{321}+a_{43}+a_{21}+a_{41}+a_{23}+a_{42}-3 a_{13}+2\right)+\ldots
\end{gathered}
$$

Again for $\xi=1$, complete polarization, the quadrangle expression (83) returns Berry's phase. 
Another easy to calculate process makes use of

$$
D^{\prime} \# D^{-1}=D^{1 / 2} D^{-1 / 2}, \quad U\left(D^{\prime}, D\right)=1_{\text {id }}, \quad \text { if } \quad D^{\prime} D=D^{\prime} D
$$

for commuting density operators.

Let us consider the following cyclic process with photons travelling in $z$-direction. The in-state $D_{1}$ is linear polarized in, say, $x$-direction with polarization degree $\xi$. Then, conserving the polarization direction, its degree is changed to $\eta$. To do this we use (84) to get $D_{2}$. In the next step the degree of polarization remains constant but the linear polarization is changed by an angle $\alpha$. We arrive at $D_{3}$. The third step consists of changing the degree of polarization back to $\xi$, leaving its direction unchanged. We obtain $D_{4}$. Finally the direction of the polarization is rotated back to the $x$-direction, so that the initial state $D_{1}$ is recovered.

To get from $D_{2}$ to $D_{3}$ we have to calculate the relative phase transporting the state along a piece of a SU(2)-orbit on the Poincaré sphere. This has been calculated in different settings in [32] and [40] yielding

$$
U\left(D_{3}, D_{2}\right)=\exp \left(-i \alpha \eta \sigma_{3}\right) .
$$

Because of (84) the phase obtained in the cyclic process, its holonomy, is computed to be

$$
U=U\left(D_{1}, D_{4}\right) U\left(D_{3}, D_{2}\right)=\exp \left[i \alpha(\xi-\eta) \sigma_{3}\right]
$$

What can be observed from the relative phase $U$ according to the formalism of quantum theory is encoded by the transition form

$$
A \rightarrow \nu(A)=\operatorname{Tr} D_{1}^{1 / 2} A D_{1}^{1 / 2} U^{*},
$$

a special form of (18), see also (19). Similar considerations can be done with spin $1 / 2$ particles and other two-level systems.

\section{Acknowledgment}

For valuable discussions and remarks I am grateful to P. M. Alberti, B. Crell, J. Dittmann, D. Petz, G. Rudolph, L. Stodolsky, H. Urbantke. I like to thank Deutsche Forschungsgemeinschaft for support under contract Al 374/1-3.

\section{REFERENCES}

[1] R. A. Fisher: Proc. Philos. Camb. Soc. 22 (1925), 700.

[2] V. Fock: Z. Phys. 49 (1928), 323.

[3] S. Kakutani: Ann. of Math. 49 (1948), 214.

[4] S. Pancharatnam: Proc. Indian Acad. Sci. A 44 (1956), 247.

[5] G. Herzberg and H. C. Longuet-Higgens: Discuss. Faraday Soc. 35 (1963), 77.

[6] S. Sakai: Bull. Am. Math. Soc. 71 (1965), 149.

[7] D. J. C. Bures: Trans. Amer. Math. Soc. 135 (1969), 199.

[8] S. Sakai: $C^{*}$-algebras and $W^{*}$-algebras, Springer, New York 1971. 
[9] H. Araki: Publ. RIMS, Kyoto Univ. 8 (1972), 335.

[10] H. Araki: Publ. RIMS, Kyoto Univ. 10 (1974), 1.

[11] W. Pusz and L. Woronowicz: Rep. Math. Phys. 8 (1975), 159.

[12] V. Cantoni: Commun. Math. Phys. 44 (1975), 125.

[13] A. Uhlmann: Rep. Math. Phys. 9 (1976), 273.

[14] S. P. Gudder, J.-P. Marchand and W. Wyss: J. Math. Phys. 20 (1979), 1963.

[15] C. A. Mead and D. G. Truhlar: J. Chem. Phys. 70 (1979), 2284.

[16] M. D. Choi: J. Operator Theory 4 (1980), 271.

[17] F. Kubo, T. Ando: Math. Ann. 246 (1980), 205.

[18] W. K. Wotters: Phys. Rev. D 23 (1981), 357.

[19] A. S. Holevo: Probabilistic and Statistical Aspects of Quantum Theory, North-Holland, Amsterdam 1982.

[20] N. N. Ćencov: Statistical decision rules and optimal inferences, Am. Math. Society, Providence 1982.

[21] H. Araki and G. A. Raggio: Lett. Math. Phys. 6 (1982), 237.

[22] G. A. Raggio: Lett. Math. Phys. 6 (1982), 233.

[23] N. Hadjisavvas: Commun. Mulh. Phys. 83 (1982), 43.

[24] P. M. Alberti: Lett. Math. Phys. 7 (1983), 25.

[25] P. M. Alberti and A. Uhlmann: Lett. Math. Phys. 7 (1983), 107.

[26] H. Kosaki: Proc. Am. Math. Soc. 89 (1983), 285.

[27] B. Simon: Phys. Rev. Lett. 51 (1983), 2167.

[28] M. V. Berry: Proc. Royal. Soc. Lond. A 392 (1984), 45.

[29] F. Wilczek and A. Zee: Phys. Rev. Lett. 52 (1984), 2111.

[30] V. Cantoni: Helvet. Phys. Acta 58 (1985), 956.

[31] A. Uhlmann: Ann. d. Physik 42 (1985), 524.

[32] A. Uhlmann: Rep. Math. Phys. 24 (1986), 229.

[33] L. L. Campbell: Proc. Am. Math. Soc. 98 (1986), 135.

[34] Y. Aharonow and J. Anandan: Phys. Rev. Lett. 58 (1987), 1593;

J. Anandan and Y. Aharonow: Phys. Rev. D 38 (1987), 1863.

[35] R. Y. Chiao and Y. S. Wu: Phys. Rev. Lett. 57 (1986), 933; M. Kitano, T. Yabuzaki, and T. Ogawa: Phys. Rev. Lett. 58 (1987), 523; D. Suter, G. Chingas, R. A. Harris and A. Pines: Mol. Phys. 61 (1987), 1327; R. Y. Chiao, A. Antaramian, K. M. Ganga, H. Jiao and S. R. Wilkinson: Phys. Rev. Lett. 60 (1988), 1214.

[36] A. Uhlmann: Parallel Transport and Holonomy along Density Operators, in Differential Geometric Methods in Theoretical Physics, eds. H. D. Doebner and J. D. Hennig, World Sci. Publ., Singapore 1987, p. 246.

[37] J. Anandan and Y. Aharonow: Phys. Rev. D 38 (1988), 1863; J. Samuel and R. Bhandari: Phys. Rev. Lett. 60 (1988), 2339; M. G. Benedict and L. Gy. Feher: Phys. Rev. D 39 (1989), 3194.

[38] L. Dąbrowski and A. Jadczyk: J. Phys. A: Math. Gen. 22 (1989), 3167.

[39] P. M. Alberti: Wiss. Z. KMU Leipzig, MNR 39 (1990), 579.

[40] L. Dąbrowski and H. Grosse: Lett. Math. Phys. 19 (1990), 205.

[41] J. Anandan: Found. Phys. 21 (1991), 1265.

[42] H. Urbantke: Am. J. Phys. 59 (1991), 503.

[43] A. Uhlmann: Lett. Math. Phys. 21 (1991), 229.

[44] V. Heinemann: Geometrie und Transformation von Paaren positiver Linearformen über $C^{*}$-Algebren, Thesis, Leipzig 1991. 
[45] P. M. Alberti: Z. Analysis Anw. 11 (1992), 293, and 11 (1992), 455.

[46] J. Dittmann and G. Rudolph: J. Math. Phys. 33 (1992), 4148.

[47] J. Dittmann and G. Rudolph: J. Geom. and Phys. 10 (1992), 93.

[48] M. Hübner: Thesis, Leipzig 1992.

[49] M. Hübner: Phys. Lett. A 163 (1992), 239.

[50] A. Uhlmann: The Metric of Bures and the Geometric Phase, in Quantum Groups and Related Topics, eds. R. Gielerak et al, Kluwer Acad. Publishers, Dordrecht 1992, pp. 267274.

[51] J. Dittmann: Seminar Sophus Lie 3 (1993), 73.

[52] A. Uhlmann: Rep. Math. Phys. 33 (1993), 253.

[53] A. Uhlmann: Parallel lifts and holonomy along density operators: Computable examples using O(3)-orbits, in Symmetry in Science VI: From the Rotation Group to Quantum Algebras, ed. B. Gruber, Plenum Publ. Co., New York 1993, pp. 741-748.

[54] M.-T. Tok: Berry-Phase, Paralleltransport und Einstein-Yang-Mills Gleichungen, Diplomarbeit, Leipzig 1993.

[55] S. L. Braunstein, C. M. Caves: Phys. Rev. Lett. 72 (1994), 3439.

[56] D. Petz: Monotone Metrics on Matrix Spaces, Linear Alg. Appl. (to appear).

[57] D. Petz, C. Sudar: Geometries of Quantum States, to appear.

[58] J. Dittmann: private communication. 